\title{
Simple Methods to Correlate Light and Scanning Electron Microscopy
}

\section{Claudia S. López, ${ }^{1,2,3 *}$ Erin Stempinski, ${ }^{1,2}$ and Jessica L. Riesterer ${ }^{1,2}$}

${ }^{1}$ Multiscale Microscopy Core, Oregon Health \& Science University (OHSU), Portland, OR

${ }^{2} \mathrm{OHSU}$ Center for Spatial Systems Biomedicine (OCSSB), Portland, OR

${ }^{3}$ Pacific Northwest CryoEM Center, Portland, OR

*lopezcl@ohsu.edu

\begin{abstract}
Based on technologies capable of data collection between the millimeter and nanometer scales, correlative imaging has been transforming how researchers obtain molecular and spatial information from specimens. Attempts to combine multidimensional data are often met with the challenge of overcoming suboptimal sample conditions such as reduced fluorescence signal, poor specimen preservation, anisotropic specimen deformation, and low specimen contrast. These issues motivated the development and use of enhanced sample preparation procedures, as well as specialized imaging software to overcome such challenges. In this work we present three simple methods to correlate optical and scanning electron microscopy images.
\end{abstract}

Keywords: scanning electron microscopy, fluorescence microscopy, correlative light and electron microscopy, focused ion beam, cancer biology

\section{Introduction}

Correlative light and electron microscopy (CLEM) can be done by means of transmission electron (TEM), scanning electron (SEM), and focused ion beam (FIB-SEM) microscopies [1-7]. In recent years, we have optimized and developed new protocols to correlate optical microscopy of fixed and live cells with both SEM and 3D FIB-SEM imaging [3,4,8-10] (unpublished results) and to also preserve fluorescence signal in methacrylate resins $[8,10]$ (unpublished results). In some experimental cases, we have observed that 2D EM imaging will suffice and is enough to answer a specific scientific question. However, over the past ten years the scientific community has shown advantages of using 3D EM. In fact, 3D imaging technologies have enormously advanced knowledge in several scientific fields, such as developmental biology, cancer biology, and, particularly, neurobiology [11-13]. In our hands, the ability to correlate both fluorescent and bright-field imaging with 3D FIB-SEM has helped us develop protocols that are currently being used in clinical trials [14-16] (https://dx.doi. org/10.17504/protocols.io.36vgre6). These protocols assist clinical pathologists and cancer biology researchers at Oregon Health and Science University to identify specific areas of interest from human cancer biopsies prepared for large format $2 \mathrm{D}$ and $3 \mathrm{D}$ EM by combining toluidine blue and SEM images (Figure 1).

An advantageous technique, sequential CLEM allows sample imaging using any optical method of choice, including super-resolution fluorescence microscopy [17], and samples are processed for 2D or 3D EM only if the optical imaging was successful. In this workflow, the introduction of the heavy metals needed to obtain high-quality images by secondary electron SEM (SE-SEM) or backscattered electron SEM (BSE-SEM) is completed in later steps to ensure that fluorescent signal is not compromised [3]. Currently, a broad range of methodologies to obtain CLEM results exists, each with their own advantages and limitations $[1,2,6,18]$. Therefore, researchers must carefully select the optimal methods for their study based on the nature of their specimens and instrumentation available. Here we describe three different CLEM methods that can be easily reproduced in any laboratory using conventional bench processing methods.

\section{Materials and Methods}

Instrumentation. Similar instrumentation and imaging conditions were used for all of the results presented here (Figure 2). Wide-field optical and fluorescence microscopy (FM) was performed using a FEI CorrSight ${ }^{\mathrm{TM}}$. A FEI Helios NanoLab 650 DualBeam ${ }^{\mathrm{TM}}$ (later upgraded to the 660 version) was used for SEM and FIB-SEM data collection. Both instruments used the FEI MAPS software package. Sections were cut using a Leica UC7 ultramicrotome. Sample coating was performed on a Leica ACE600 coater and dehydration using a Leica CPD300.

There are several software programs, both open-source or licensed, available for optical and EM correlation that include rigid (MAPS used here from Thermo Fisher) and/or non-rigid registrations of the images [19]. Depending on the protocol used to prepare the specimen, non-rigid registration is advantageous as the image can be warped and therefore adapted to any deformations that have occurred during the preparation steps (mostly during the chemical fixation and dehydration procedures). However, for samples processed near to native conditions, such as those methods used for cryofixation, or in the case of in-resin fluorescence preservation methods, rigid registration is most appropriate since specimen deformation is minimal.

Correlation of optical microscopy with 2D SE-SEM imaging. MCF7 breast cancer cells (American Type Culture Collection) were grown in Dulbecco's modified Eagle's medium (DMEM) supplemented with $10 \%$ fetal bovine serum (FBS) in $35 \mathrm{~mm}$ culture dishes. Cells were transiently cotransfected with 500 ng of DNA of HER2-eGFP and AKT2-tagRFP expression plasmids each using X-tremeGENE HP DNA Transfection Reagent (Roche) for $24 \mathrm{~h}$. Cells were grown on indium tin oxide (ITO)-coated cover slips pretreated with $0.01 \%$ polylysine at a desired confluency and chemically fixed using $4 \%(\mathrm{v} / \mathrm{v})$ paraformaldehyde (PFA) and $0.1 \%(\mathrm{v} / \mathrm{v})$ glutaraldehyde (Glut) in $1 \times$ phosphate-buffered saline (PBS) for $1 \mathrm{~h}$ at room temperature. After this step, the cells were washed in $1 \times$ PBS and incubated with $1 \mu \mathrm{g} / \mathrm{ml}$ DAPI prepared in $1 \times$ PBS and incubated for 30 minutes at room temperature. Before acquiring the optical images, the ITO cover slips were engraved using 

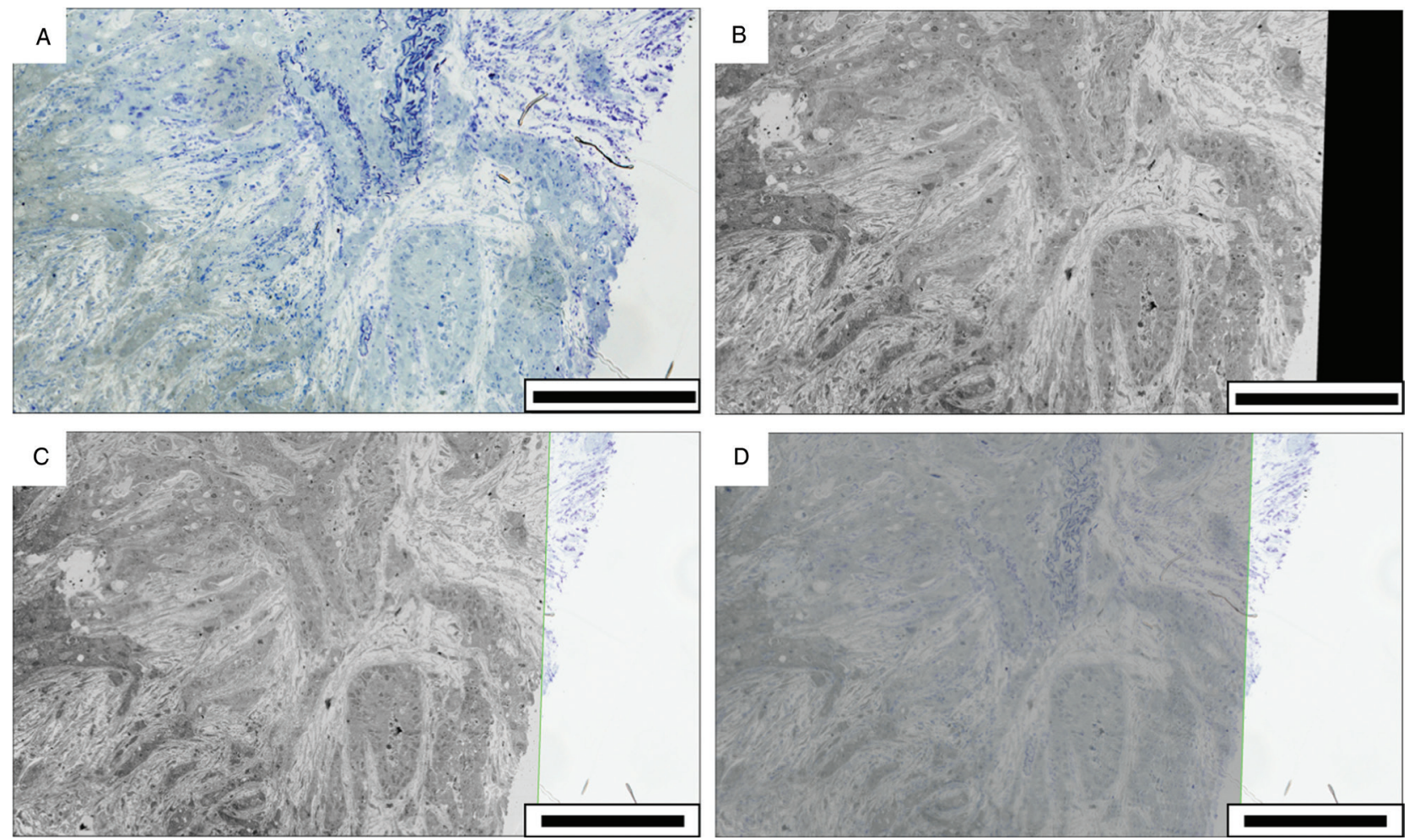

Figure 1: Specific regions of interest of pancreatic ductal adenocarcinoma (PDAC) pointed out by pathologists can be studied at higher resolution using CLEM. The Toluidine Blue stained thick section in (A) can be matched to the SEM image montage in (B). By changing the overlaid image opacity from $100 \%$ in (C) to $50 \%$ in (D), specific cells can be identified in both modalities. Scale bars are equal to $200 \mu \mathrm{m}$.

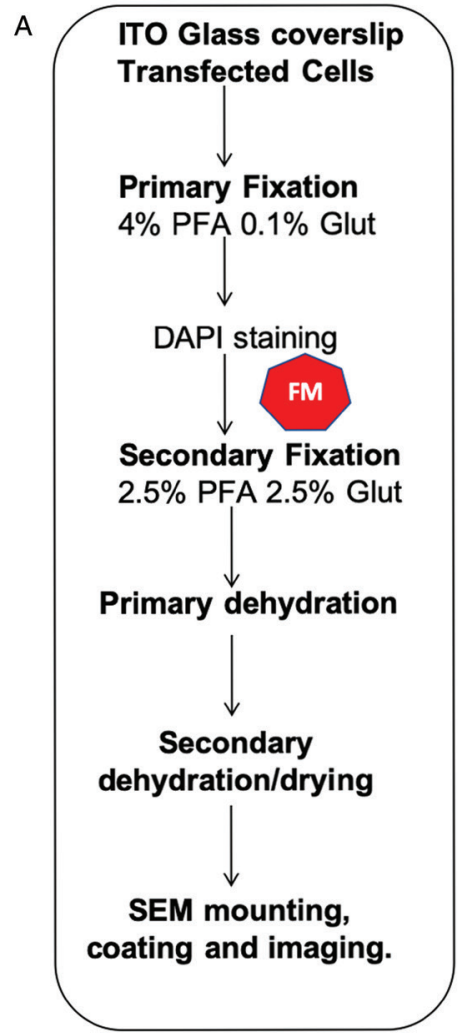

B

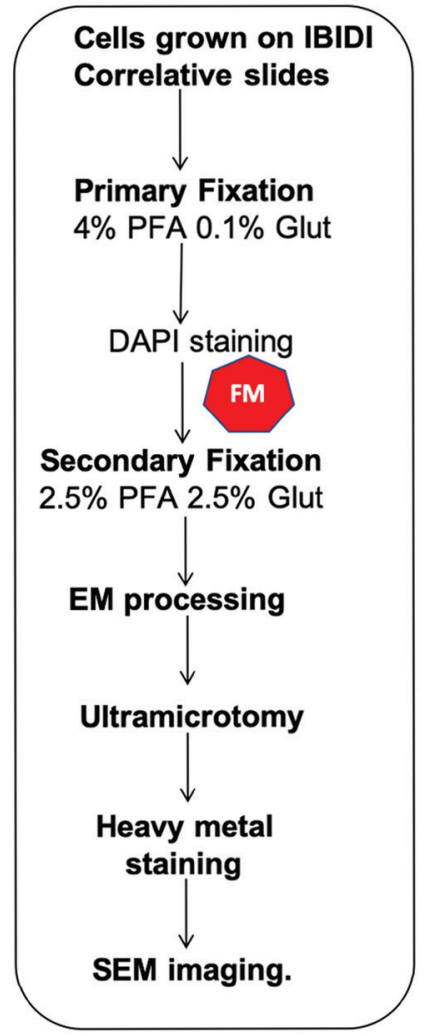

C

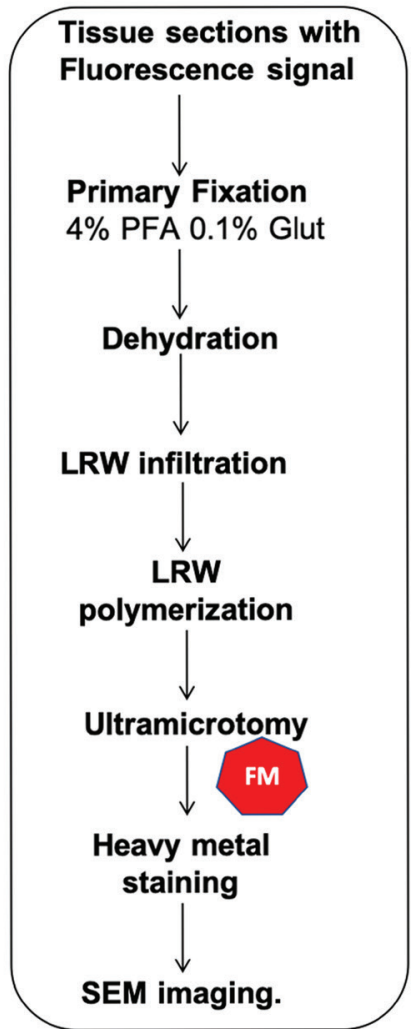

Figure 2: CLEM workflows described in this manuscript. (A) FM and 2D SE-SEM, (B) FM and 2D-BSE-SEM, (C) FM and 2D-SEM in resin fluorescence preservation. The symbol in red indicates the FM step. 
a diamond scribe as described previously [9]. Cells were imaged by optical microscopy using the CorrSight fluorescence microscope. Tile sets of increasing magnifications were recorded, starting at $5 \times$. Based on the overview tile set, higher resolved images at $20 \times, 40 \times$, and $63 \times$ were acquired, narrowing down the regions of interest (ROI) to single cells [3]. After this step the specimen was ready to be processed for SEM. ITO cover slips were transferred to a strong Karnovsky's fixative [20] for $1 \mathrm{~h}$ at room temperature, washed with water, and dehydrated using an increasing concentration series of ethanol: $\mathrm{H}_{2} \mathrm{O}$ solutions up to $100 \%$ ethanol [9]. Samples were then subjected to critical point drying using a Leica CPD 200. After drying, cells were carbon coated $(3-5 \mathrm{~nm})$ before being mounted on conventional SEM pins using silver paint. SE-SEM images were collected using an Everhart-Thornley (ETD) or through lens (TLD) detector. MAPS was used to correlate the optical images to the SEM images as described previously [3]. Note: Incubation of specimens in $2 \% \mathrm{OsO}_{4}$ prior to the dehydration step helps to minimize charging of the cells in the SEM.

Correlation of optical microscopy with 2D BSE-SEM imaging. MCF7 breast cancer cells were grown as described above on IBIDI correlative slides and chemically fixed, washed, and incubated using the same solutions and procedures as described above. Cells were imaged by wide-field optical microscopy. After this, the specimen was processed for BSESEM, described elsewhere [3] (https://dx.doi.org/10.17504/ protocols.io.36vgre6). Using the imprinted IBIDI grid on the resulting Epon block as a reference, 150-250 nm thick ultramicrotome sections from specific ROIs were generated and mounted on ITO cover slips or $5 \times 5 \mathrm{~mm}$ silicon chips. The directional backscatter detector (DBS) was used to image plastic sections for BSE-SEM.

In-resin (methacrylate) fluorescence preservation and 2D BSE-SEM imaging. London resin white (LRW) was used to preserve fluorescence signal of KMC mouse pancreas injected with Cisplatin labeled with tetramethylrhodamine (TRITC) [21]. Tissue sections were fixed in 4\% PFA only for $24 \mathrm{~h}$ and incubated post-fixation for $2 \mathrm{~h}$ in $0.5 \mathrm{M}$ Tris $\mathrm{pH} 7.2,0.1 \mathrm{M}$ glycine to reduce autofluorescence. Tissue sections were then dehydrated in a concentration series of ethanol: $\mathrm{H}_{2} \mathrm{O}$ on ice up to $90 \%$ ethanol: $\mathrm{H}_{2} \mathrm{O}$. After this step the specimens were infiltrated with solutions consisting of LRW and 90\% ethanol (1:2, 1:1), respectively, for $1 \mathrm{~h}$ on ice. Specimens were then infiltrated overnight in 100\% LRW at $20^{\circ} \mathrm{C}$. The next day, the LRW was exchanged with fresh LRW once, and samples were polymerized at $50^{\circ} \mathrm{C}$ for $24 \mathrm{~h}$ in a vacuum oven. Sections (150-250 nm-thick) were cut and mounted on ITO cover slips. Plastic sections were imaged on the CorrSight using the shuttle holder and a triple-band filter set. After this, the sections were stained for 3 minutes with Reynolds' lead citrate, washed extensively with $\mathrm{H}_{2} \mathrm{O}$, and then stained for 3 minutes with $5 \% \mathrm{w} / \mathrm{v}$ uranyl acetate prepared in $\mathrm{H}_{2} \mathrm{O}$. Sections were washed again with $\mathrm{H}_{2} \mathrm{O}$. Samples were air-dried before again mounting on the shuttle holder for SEM imaging.

\section{Results and Discussion}

Correlation of fluorescence signal with 2D SE-SEM imaging. Figure 2A shows the sequential CLEM workflow used to image samples by FM and SE-SEM. SE-SEM imaging was used here to provide information of the topographic characteristics of cells under study. The protocol discussed here is very simple and can be easily reproduced in any institution with an EM core facility. In this work, $0.01 \%$ poly-l-lysine pretreated ITO cover slips were used for cell growth, but any other matrix can be used. Disturbed cellular growth behavior was not observed by the use of ITO cover slips, but researchers must monitor their specific experiment.

Finding the same ROI across imaging platforms is the most difficult part of CLEM. The advantage of the MAPS software for image acquisition is that all positional information is recorded, helping the user localize the same ROI on the SEM. In addition, images acquired from any optical microscope can be imported for correlation. The addition of fiducials is essential and will facilitate the relocation of the ROI. In the example included here, we marked the ITO cover slip using a diamond scribe [9]. This mark was visible on both imaging platforms. More elegant fiduciary markers can be used, such as pre-labeled cover slips or fluorospheres, but are not necessary.

Once the fluorescence imaging is finished, a secondary fixation using strong fixatives at higher concentration is used (Figure 2A). As mentioned previously, the dehydration steps were done using an increasing ethanol: $\mathrm{H}_{2} \mathrm{O}$ series up to $100 \%$ ethanol, and the final drying step was done using a critical point dryer. These steps are crucial and must be done carefully in order to minimize sample shrinkage. For this step we recommend a slow increase of ethanol concentration, avoid removing the slides from the solutions to prevent air drying the specimen, and a slow purging of ethanol in the critical point dryer. It is also important to use fresh solvents to minimize the water content of the final dehydration solution, otherwise dehydration defects can be introduced to the specimen. Figure 3 shows an example of cells dehydrated rapidly, resulting in an excessive shrinking of the cellular body and filopodia. In this example the FM image cannot be perfectly overlaid with the SE-SEM image by using rigid registration methods.

In Figure 4A, a $5 \times$ tiled image shows an overlay of brightfield images and FM of the cells grown on the ITO cover slip. As proof of concept, in this experiment we used the overlay of fluorescence imaging onto SE-SEM images to localize proteins, such as human epidermal growth factor receptor 2 (HER2), that play an important role in cancer metastasis. HER2 overexpression is present in some types of breast cancer cases and is associated with poor prognosis [22]. As expected the HER2-GFP signal is mostly located at the plasma membrane (Figures 4B and 4D). AKT2-RFP, a beta serine/threonine-protein kinase, is observed in the red channel and is mostly distributed in the cytoplasm. DAPI staining allowed visualization of nuclear DNA in the cells and could also be used to determine the number of nuclei and assess gross nuclear morphology (Figure 4B). Figures $4 \mathrm{C}$ and $4 \mathrm{D}$ show missing cells between FM and SEM imaging as well as anisotropic shrinkage between the cells. The overlay fitting was optimized for the central cell showing HER2 signal. SEM images were collected with the ETD detector at $1 \mathrm{keV}$ and $50 \mathrm{pA}$.

Correlation of fluorescence microscopy with 2D BSESEM imaging on Epon plastic section. We have used IBIDI correlative slides to develop the 2D CLEM method described here [3]. Our main goal was to develop this CLEM method to 


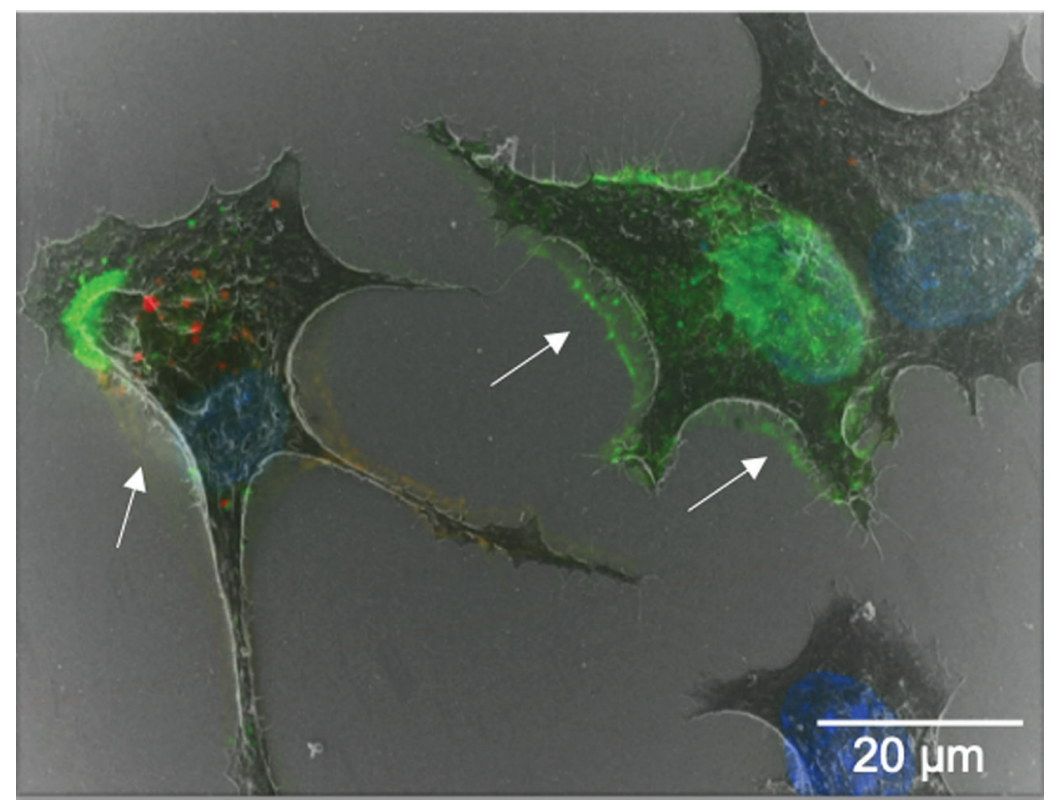

Figure 3: Cell shrinkage and distortion generated during dehydration steps. FM and SESEM overlay of cells grown on ITO cover slips. Blue: DAPI, Green: HER2-GFP, Red: AKT2-RFP. characterize the morphology, number, and localization of nucleoli in cultured breast cancer cells. The presence of prominent nucleolar structures in tumor tissues is one of the parameters used by pathologists to define the "nuclear grade of tumors" [23]. Moreover, larger nucleolar size is recognized as a contributor in both tumor initiation and cancer progression [24]. In this example, the fiducials used for correlation were the grids imprinted in the wells where cells were grown. Cells grown on these IBIDI slides were primary fixed as described above and imaged by FM (Figure 1B). However, as we have described elsewhere [3], live cell imaging can also be performed using this substrate. During FM screening, the absence of DAPI staining within the nuclei of the cells revealed the localization of the nucleoli (Figures 5A and D). Based on the ROI locations and using the IBIDI grid that is visible on the block under any optical microscope as reference, we generated $250 \mathrm{~nm}$ thick sections after staining and embedding the cells. As shown in the image overlays (Figures 5C and F), the absence of DAPI signal shown in Figures $5 \mathrm{~A}$ and $5 \mathrm{D}$ provides an indication of nucleoli presence in the BSE-SEM

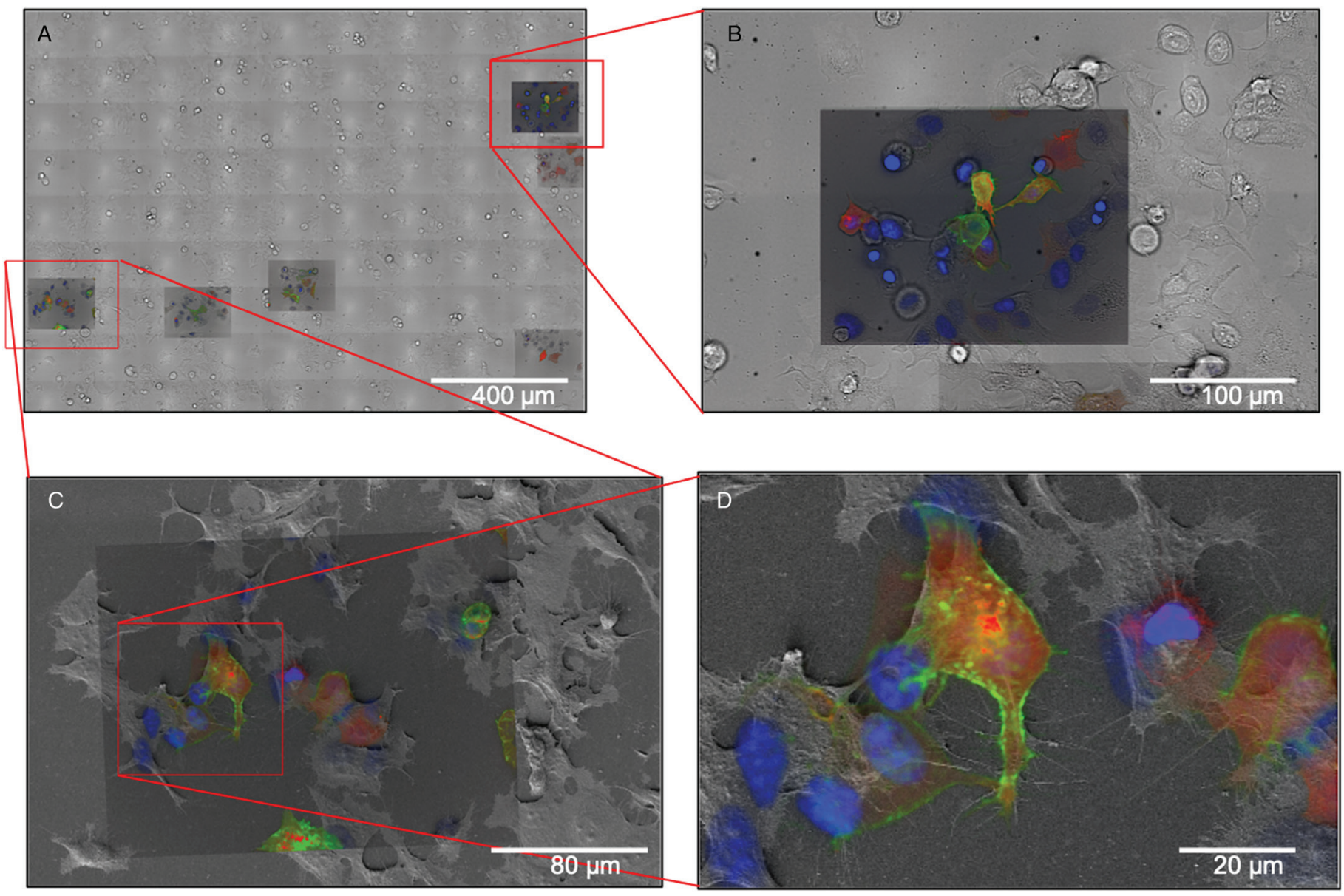

Figure 4: MFC7 breast cancer cells grown on ITO cover slips and labeled for membrane, cellular, and nuclear markers. (A) 20× brightfield with fluorescence signal overlays. (B) 40× GFP and RFP tagged proteins together with DAPI signal overlayed with transmitted light. (C) 40× fluorescence signal overlayed with the SE-SEM. (D) Higher magnification of labeled region of interest showing more cellular details. Scale bars: A: $400 \mu \mathrm{m}, \mathrm{B}: 100 \mu \mathrm{m}, \mathrm{C}: 80 \mu \mathrm{m}, \mathrm{D}: 20 \mu \mathrm{m}$. Images show missing cells between FM and SEM due to sample preparation as well as anisotropic shrinkage. 

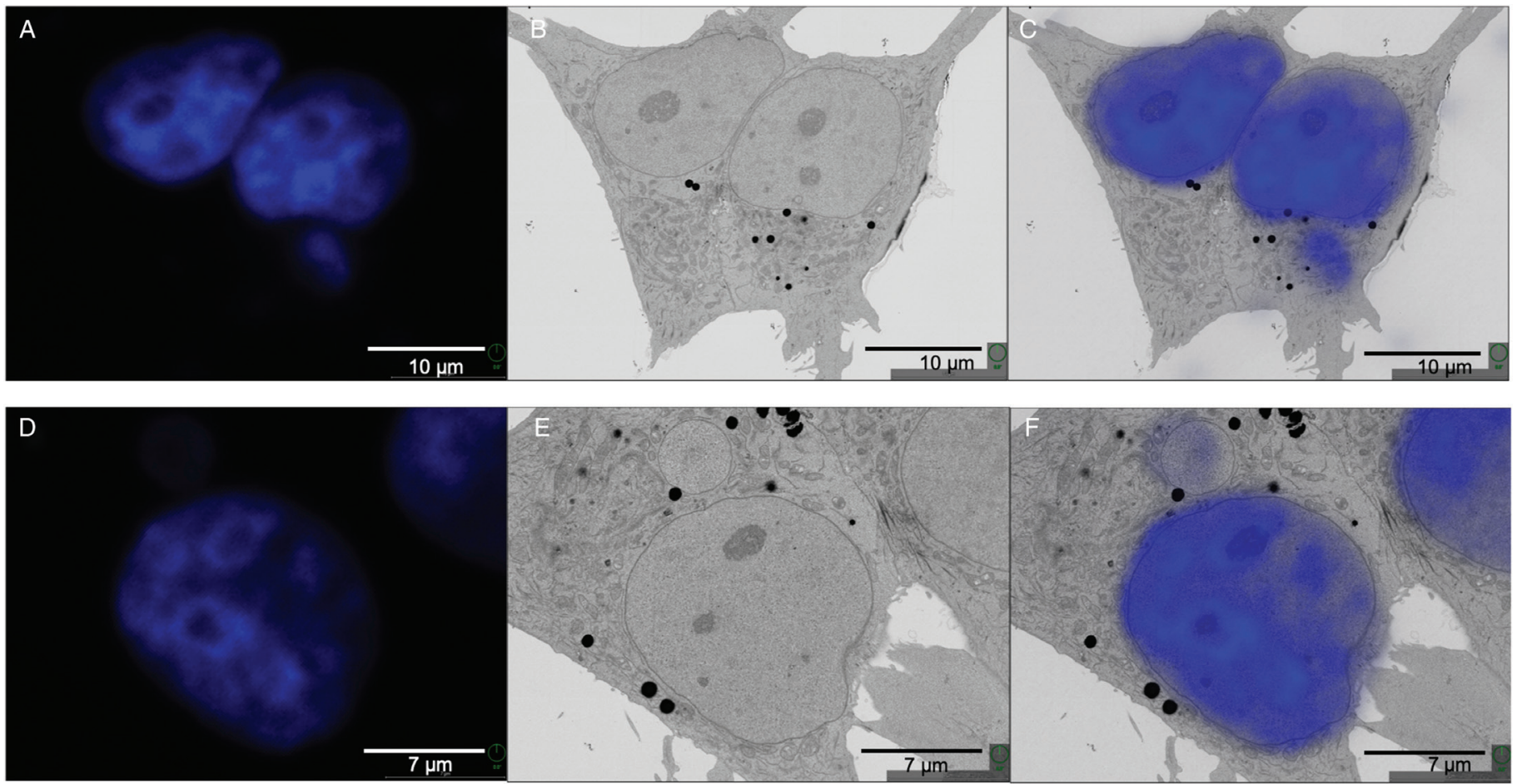

Figure 5: (A) and (D) Representative images of DAPI stained cells showing cellular nucleoli in the areas voided of DAPI signal. (B) and (E) BSE-SEM images of the same cells. (C) and (F) FM and BSE-SEM overlays created using MAPS 3.1. Scale bars: A, B, and C: $10 \mu \mathrm{m}$; D, E, and F: $7 \mu \mathrm{m}$.

images. BSE-SEM images were acquired using $2.5 \mathrm{keV}, 0.2 \mathrm{nA}$, and the DBS detector. Although here we only show the correlation between $2 \mathrm{D}$ images, the same samples were subjected to 3D FIB-SEM to study the ultrastructural aspects of the nucleoli region on these cells (unpublished results).

In-resin fluorescence preservation and correlation with BSE-SEM imaging. As described in the Materials and Methods section, the Figure 1C protocol was performed at low temperatures, and the dehydration process proceeded up to $90 \%$ ethanol to preserve fluorescence signal from the Cisplatin-TRITC in the mouse pancreas. Keeping 10\% water content in the specimen helps preserve the signal of the fluorophore most likely due to the maintenance of a hydration shell around it. Figure 6A shows a representative image of the fluorescence signal obtained from the LRW sections. Although there is some autofluorescence background present in the tissue, there are specific areas in the specimen showing higher signal intensity, more specifically on certain cells located near pancreatic ducts. Figure 6B shows a low-magnification SEM image of the same field of view of the image shown in Figure 6A after heavy metal staining. Here we used the shape of the plastic section to correlate the images as well as features present in the tissue (that is, ducts). Figure $6 \mathrm{C}$ shows a higher-magnification image from a specific region boxed in Figure 6B. One of the cells showing TRITC signal near the duct can be observed. Although we only used mild chemical fixation methods to fix this specimen, cellular membranes and cellular structures were well preserved.
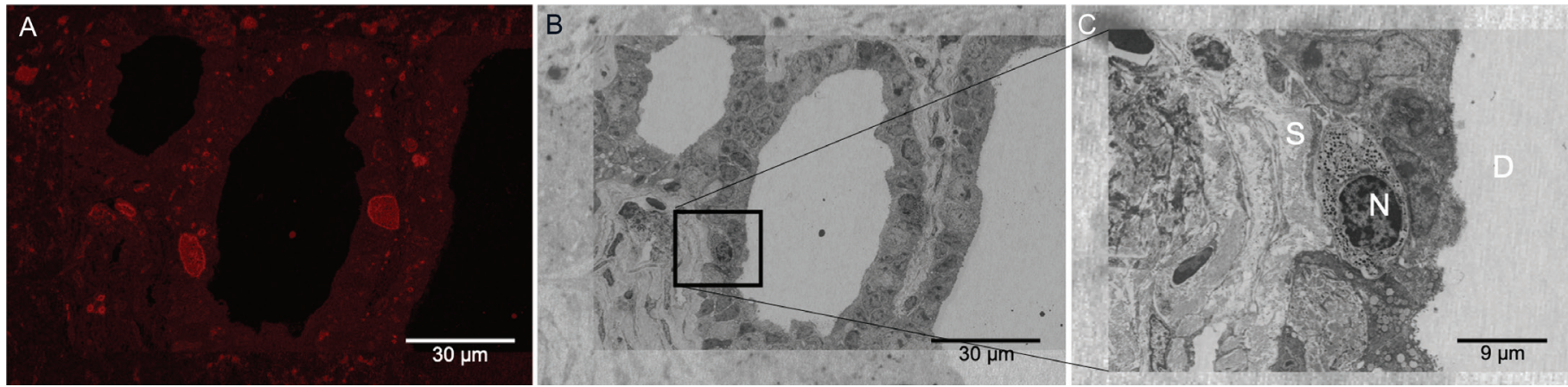

Figure 6: LRW plastic section showing Cisplatin-TRITC fluorescence preservation in mouse pancreas. (A) Pancreatic duct showing fluorescent positive acinar cells. (B) Same area as in (A) acquired by BSE-SEM imaging. The area boxed in black corresponds to an acinar cell showing fluorescence signal. (C) Higher magnification of the boxed area in (B) is shown. Secretory granules are identifiable in the acinar cells. N: nucleus, S: stroma, D: duct. 


\section{Conclusion}

The presence of internal or external fiducial markers is very important for any sequential CLEM workflow. These markers can be either added to the specimen (gold fiducials, nanospheres, etc.), be part of the specimen (features within the sample, shape, etc.), or embedded within the sample holder or substrate of choice $[1,4,5,7]$. In the work described here, the three CLEM procedures used were sequential and, in each case, a different fiducial marker was exploited (diamond scribed marks, embedded grids on epoxy blocks, and plastic section shaped together with tissue morphology). Although our imaging used commercial correlative software, the workflows described here can be replicated using any instrument since the fiducials described are visible in both imaging platforms and should help the researcher locate the ROIs. That being said, a correlative image software program is essential for bridging the differences in resolution and enabling the integration of molecular and structural information.

\section{Acknowledgements}

Microscopy was performed at the Multiscale Microscopy Core, a University Shared Resource at OHSU. The authors acknowledge funding from OCSSB to CSL and NIH-NCI Cancer Center Support Grant (CCSG) 2P30CA069533. The Brenden-Colson Center for Pancreatic Care additionally funded portions of this work. We would also like to acknowledge all the collaborators who contributed with samples and advice.

\section{References}

[1] T Muller-Reichert et al., Methods Cell Biol 124 (2014) xviixviii.

[2] T Muller-Reichert and P Verkade, Methods Cell Biol 111 (2012) xvii-xix.

[3] CS Lopez et al., Methods Cell Biol 140 (2017) 149-64.

[4] JK Doh et al., Proc Natl Acad Sci US 115 (2018) 12961-66.

[5] CJ Peddie et al., Ultramicroscopy 143 (2014) 3-14.

[6] CJ Peddie et al., Methods Cell Biol 124 (2014) 363-89.

[7] J Kuipers et al., Cell Tissue Res 360 (2015) 61-70.

[8] D Keene et al., Microsc Microanal 22 (2016) 206-07.

[9] JK Doh et al., Bio-protocol 9 (2019) e3414.

[10] A Schaser et al., Microsc Microanal 25 (2019) 1132-33.

[11] LK Scheffer and IA Meinertzhagen, Ann Rev Cell Devel Biol 35 (2019) 637-53.

[12] CJ Peddie and LM Collinson, Micron 61 (2014) 9-19.

[13] A Kremer et al., J Microsc 259 (2015) 80-96.

[14] B Johnson et al., Cancer Res 78 (2018) 3296.

[15] JL Riesterer et al., Microsc Microanal 25 (2019) 1194-95.

[16] JL Riesterer et al., Methods Cell Biol 158 (2020) 163-81.

[17] Y Zhang et al., PLoS One 12 (2017) e0176839.

[18] P de Boer et al., Nat Methods 12 (2015) 503-13.

[19] P Paul-Gilloteaux et al., Nat Methods 14 (2017) 102-03.

[20] MJA Karnovsky, J Cell Biol 27 (1965) 137-38A.

[21] PM Uribe et al., PLoS One 8 (2013) e55359.

[22] SJ Slamon et al., Science 235 (1987) 177-82.

[23] M Derenzini et al., Histopath 54 (2009) 753-62.

[24] I Orsolic et al., Semin Cancer Biol 37-38 (2016) 36-50.

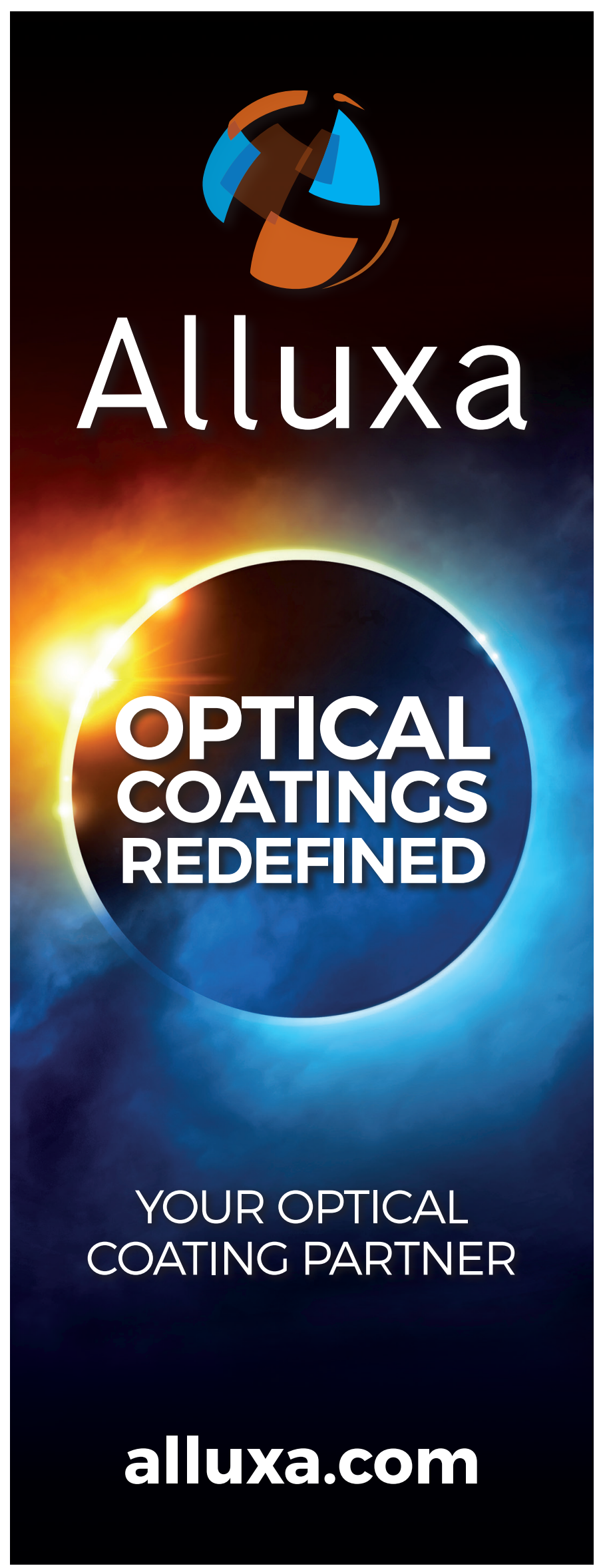

Microscopytoday 